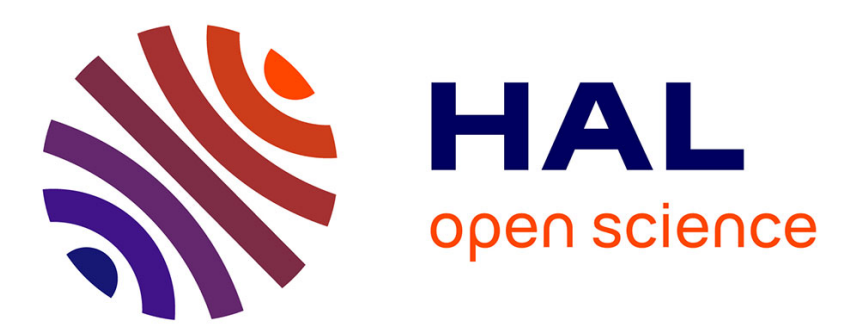

\title{
L'adoption plénière en France et en Angleterre-Galles : une histoire comparée du droit et des pratiques (1926-2015) \\ Jean-François Mignot
}

\section{- To cite this version:}

Jean-François Mignot. L'adoption plénière en France et en Angleterre-Galles : une histoire comparée du droit et des pratiques (1926-2015). Droit et Société, 2016, 93 (2), pp.417-435. halshs-01348782

\section{HAL Id: halshs-01348782 \\ https://shs.hal.science/halshs-01348782}

Submitted on 25 Jul 2016

HAL is a multi-disciplinary open access archive for the deposit and dissemination of scientific research documents, whether they are published or not. The documents may come from teaching and research institutions in France or abroad, or from public or private research centers.
L'archive ouverte pluridisciplinaire HAL, est destinée au dépôt et à la diffusion de documents scientifiques de niveau recherche, publiés ou non, émanant des établissements d'enseignement et de recherche français ou étrangers, des laboratoires publics ou privés. 


\title{
L'adoption plénière en France et en Angleterre-Galles : une histoire comparée du droit et des pratiques (1926-2015)
}

\author{
Jean-François Mignot
}

Groupe d'Étude des Méthodes de l'Analyse Sociologique de la Sorbonne (GEMASS), 20 rue Berbier-du-Mets F-75013 Paris.

<jean-francois.mignot@cnrs.fr>

Résumé

Pourquoi les Britanniques adoptent-ils très peu d'enfants à l'international, par rapport aux Français? Pour le savoir, nous comparons l'histoire du droit et des pratiques de l'adoption plénière dans les deux pays. Il apparaît alors que, depuis l'entre-deuxguerres, les deux pays ont des droits de l'adoption proches, mais qu'en AngleterreGalles les mineurs nationaux maltraités sont beaucoup plus souvent placés pour l'adoption qu'en France. Cette différence majeure a eu des conséquences importantes sur la pratique de l'adoption dans ces deux pays. D'une part, les adoptions ont longtemps été beaucoup plus nombreuses en Angleterre-Galles qu'en France. Et, d'autre part, les pouvoirs publics britanniques sont avant tout soucieux de faire adopter leurs nombreux mineurs nationaux, notamment, depuis les années 1970, de manière «ouverte ». C'est pourquoi, en Angleterre-Galles, si peu de mineurs étrangers sont adoptés.

Adoption ouverte et plénière - Droit comparé - Famille - Intérêt de l'enfant - Mineur maltraité - Statistiques.

\section{Summary Adoption in England-Wales and France : A Compared History}

Why do the British adopt so few children abroad, compared to the French? To better understand this difference, I compare the history of adoption law and adoption practices in England-Wales and in France. Since the 1920s both countries have had similar adoption laws, but abused children have been placed for adoption much more often in England-Wales than in France. This major difference has had important consequences on the practice of adoption in these two countries. First, for a long time the number of adoptions has been much higher in England-Wales than in France. Second, British governments aim at having their numerous adoptable children adopted, including through open adoption since the 1970s. This is why English people adopt so few foreign children.

Abused child - Best interest of the child - Comparative law - Family - Open adoption-Statistics. 


\section{Introduction}

Les populations de la France et du Royaume-Uni sont à peu près de même taille, et leurs comportements démographiques sont pour la plupart similaires. Toutefois, alors que la France est l'un des pays où, depuis les années 1970, on adopte le plus grand nombre d'enfants à l'international, tel n'est pas le cas du Royaume-Uni, où l'on en adopte même moins que dans certains pays beaucoup plus petits comme les Pays-Bas, la Suède ou la Norvège ${ }^{1}$. Cette différence de comportements s'explique-t-elle par une différence entre les droits français et britannique de l'adoption, ou d'autres facteurs sont-ils en jeu ? Pour le savoir, nous proposons une histoire comparée du droit et des pratiques de l'adoption entre la France et le Royaume-Uni. Notre analyse mêle ainsi le droit, la démographie et la sociologie, dans une perspective historique. À notre connaissance, il s'agit du premier article cherchant à comparer l'histoire de l'adoption en France et au Royaume-Uni, même s'il existe déjà plusieurs études comparatives de l'adoption entre la France et un autre pays anglo-saxon - mais il s'agit du pays qui, de très loin, adopte le plus d'enfants à l'international : les États-Unis 2 .

Plus précisément, l'article compare la France non pas au Royaume-Uni mais à la seule Angleterre-Galles. En effet, cette partie du Royaume-Uni, qui est soumise au même droit de l'adoption (un droit qui diffère de ceux en vigueur en Écosse ou en Irlande du Nord), est aussi la seule partie du pays sur laquelle on dispose de données empiriques sur le long terme. En outre, l'article compare les adoptions en Angleterre-Galles non pas à toutes les adoptions en France, mais aux seules adoptions plénières. Il s'agit d'une institution juridique connue dans la plupart des pays du monde ${ }^{3}$, dont la France et l'Angleterre-Galles, et qui crée, entre un mineur adopté et un couple ou un individu adoptant, un lien de filiation qui remplace le lien originel entre le mineur adopté et ses parents biologiques. L'adoption plénière crée ainsi un lien nouveau, substitutif et exclusif entre l'adopté et l'adoptant, en conséquence de quoi l'adopté hérite son nom et ses droits de succession de son seul adoptant. Par contraste, l'adoption simple, qui existe dans certains pays de droit germano-romain codifié comme la France, mais qui n'existe pas dans les pays de common law comme le Royaume-Uni ${ }^{4}$, ne crée entre l'adopté - souvent majeur - et l'adoptant qu'un lien de filiation qui s'ajoute à celui existant déjà entre l'adopté et ses parents biologiques. Étant donné que l'Angleterre-Galles ne connaît pas l'adoption simple, et que cette institution a généralement une visée très différente de l'adoption plénière (une visée successorale plutôt qu'éducative), nous ne comparons entre elles que les adoptions plénières 5 . Enfin, l'article n'aborde pas dans le détail l'adoption par les couples homosexuels : sa légalisation est trop récente pour que l'on dispose à ce sujet de données

1. Peter Selman, « Global Trends in Intercountry Adoption: 2001-2010 », Adoption Advocate, 44, 2012, p. 1-17.

2. Ivan JABLONKA, «L'adoption aux États-Unis et en France ( $\mathrm{XIX}^{\mathrm{e}}-\mathrm{XX}^{\mathrm{e}}$ siècles) », Histoire et sociétés, 15 (3), 2005, p. 56-65 ; Valérie DOUMENG, «Étude comparative de l'adoption française et de l'adoption ouverte aux États-Unis », in Agnès Fine et Claire NEIRINCK (dir.), Parents de sang, parents adoptifs. Approches juridiques et anthropologiques de l'adoption. France, Europe, USA, Canada, Paris : LGDJ, 2000, p. 147-167; Sylvie SAGNES, «La revanche élective. Filiation adoptive et "origines" en France et aux États-Unis », in Agnès FinE et Claire NEIRINCK (dir.), Parents de sang, parents adoptifs, op. cit., p. 169-183.

3. Nations Unies, Child Adoption: Trends and Policies, New York : United Nations Publications, 2009.

4. Carmen LavalleE, « Pour une adoption sans rupture du lien de filiation d'origine dans les juridictions de civil law et de common law », Informations sociales, 146 (2), 2008, p. 132-140.

5. Sur l'adoption simple, voir Pierre VERDIER, «Limited Adoption in France », Adoption \& Fostering, 12 (1), 1988, p. 41-44. 
empiriques comparables (loi du 17 mai 2013 qui ouvre le mariage aux couples de personnes de même sexe en France, et Adoption and Children Act de 2002 qui ouvre l'adoption aux couples non mariés en Angleterre-Galles).

\section{Des histoires du droit de l'adoption globalement similaires}

Depuis quand l'adoption est-elle connue des droits français et britannique ? Qui peut adopter, qui peut être adopté, selon quelle procédure et avec quels effets juridiques ? Pour le savoir, comparons les histoires du droit de l'adoption en France 6 et en Angleterre-Galles 7.

\section{I.1. Jusqu'à la Première Guerre mondiale}

L'adoption plénière n'est introduite dans les droits français et britannique qu'à partir de l'entre-deux-guerres. En effet, même si jusqu'à la Première Guerre mondiale des couples stériles recueillent des mineurs orphelins ou abandonnés, ces démarches restent informelles : aucun dispositif juridique ne permet à un couple marié, ou a fortiori à une personne agissant seule, d'établir avec un mineur étranger à sa famille un lien de filiation substitutif et exclusif. Ainsi, aucun des célèbres enfants orphelins ou abandonnés du roman du XIX ${ }^{\mathrm{e}}$ siècle n'est adopté : en France, ni Cosette des Misérables de Victor Hugo (1862), ni Rémi de Sans famille d'Hector Malot (1878), ni le fils Vallin d'Aux champs de Guy de Maupassant (1882); en Angleterre, ni Fanny Price de Mansfield Park de Jane Austen (1814), ni Jane Eyre de Charlotte Brontë (1847), ni les orphelins Oliver Twist (1839), David Copperfield (1850) ou Pip des Grandes espérances (1860) de Charles Dickens. Comme l'institution juridique de l'adoption n'existe pas, les parents d'accueil d'un mineur courent toujours le risque que ses parents biologiques demandent à en reprendre la garde. En France, le Code civil de 1804 a bien introduit l'adoption en droit, mais jusqu'à la loi du 19 juin 1923 la seule adoption qui existe est une adoption simple de majeurs, à visée successorale. En Angleterre-Galles, la common law ne connaît pas non plus l'adoption plénière. Jusqu'à l'Adoption of Children Act de 1926, l'adoption contredit l'inaliénabilité des droits et devoirs parentaux ainsi que les règles coutumières de succession. Les mesures de protection de l'enfance qu'adoptent la France et l'Angleterre à partir de la fin du XIX ${ }^{\mathrm{e}}$ siècle n'incluent donc aucunement l'adoption.

Si l'adoption plénière n'est introduite que tardivement, c'est en partie en raison de la méfiance que suscite l'adoption d'un mineur abandonné, qui est très vraisemblablement né illégitime. Qui pourrait vouloir d'un enfant qui, «né du péché », a toutes les chances de «porter le vice en lui $\gg 8$ ? Et qui pourrait vouloir que sa famille soit associée à la honte de l'illégitimité ? Dans ce contexte, c'est l'explosion du nombre d'orphelins de guerre - pour la

6. Jean-Pierre Gutton, Histoire de l'adoption en France, Paris : Publisud, 1993 ; Juliette HaLIFaX, L'adoption plénière en France : de l'établissement d'une filiation légale à la constitution d'une filiation sociale, thèse de démographie, Lille : Atelier national de reproduction des thèses, 2007 ; Claire NEIRINCK, «L'évolution de l'adoption », in Agnès FinE et Claire NEIRINCK (dir.), Parents de sang, parents adoptifs, op. cit., p. 343-361.

7. Jacqueline Flauss-DiEM, « Angleterre », Revue internationale de droit comparé, 37 (3), 1985, p. 539-556; Jenny KeAting, A Child for Keeps: The History of Adoption in England, 1918-1945, Basingstoke : Palgrave Macmillan, 2008 ; CENTER FOR ADOPTION POLICY, «Overview of English Adoption Law», 2014. Disponible à l'adresse suivante: 〈http://www.adoptionpolicy.org/pdf/eu-england.pdf>.

8. Sur la suspicion avec laquelle est encore aujourd'hui regardée l'adoption, voir Allen P. FISHER, «Still "Not Quite As Good As Having Your Own"? Toward a Sociology of Adoption », Annual Review of Sociology, 29 (1), 2003, p. 335361 . 
plupart légitimes - suite à la Première Guerre mondiale et ses 1400000 morts en France et 800000 morts au Royaume-Uni ${ }^{9}$ qui conduit les législateurs français et britannique à institutionnaliser l'adoption. De ce point de vue, d'autres pays occidentaux ont été plus précurseurs. Les États-Unis sont le premier pays occidental à introduire dans leur droit l'adoption plénière, par An Act to Provide for the Adoption of Children au Massachusetts en 1851, puis par d'autres lois dans chacun des autres États fédérés jusqu'à la décennie 1870. Des lois similaires sont ensuite adoptées en Nouvelle-Zélande (1881), en Australie-Occidentale (Australie, 1896) et en Colombie-Britannique (Canada, 1920). La plupart des pays de common law ont donc adopté des législations modernes de l'adoption avant les pays de droit romano-germanique.

\section{I.2. De l'entre-deux-guerres au tournant des années 1960 et 1970}

L'adoption plénière est introduite en Angleterre-Galles par l'Adoption of Children Act de 1926 (puis en Irlande du Nord en 1929 et en Écosse en 1930). Suite à la Première Guerre mondiale, des associations privées à but non lucratif commencent à organiser le placement de mineurs dans des familles d'accueil stables, de la bonne société. Parmi ces associations figurent la National Child Adoption Association (NCAA), la National Adoption Society (NAS) et le National Council for the Unmarried Mother and her Child (NCUMC), qui dès le début des années 1920 militent en faveur d'une loi qui organiserait juridiquement l'adoption de mineurs. Leur objectif est qu'une loi permette l'adoption dans l'intérêt supérieur de l'enfant et que les associations qui font office d'intermédiaires respectent certaines règles communes, quitte à contrarier les intermédiaires peu scrupuleux qui recueillent des enfants et les vendent pour adoption (baby farming). L'Adoption of Children Act de 1926 comporte plusieurs dispositions importantes. D'une part, sauf exceptions, un mineur ne peut devenir adoptable qu'avec le consentement de ses parents d'origine. D'autre part, peuvent adopter non seulement les couples mariés, mais aussi les individus majeurs agissant seuls, sans condition supplémentaire. Enfin, l'adoption substitue à l'ancien lien de filiation entre l'adopté et sa famille d'origine un lien, nouveau, avec sa famille adoptive. Cela dit, l'adopté n'hérite pas comme le ferait un enfant légitime avant l'Adoption of Children Act de 1949.

Alors que, pendant l'entre-deux-guerres et jusqu'à la Seconde Guerre mondiale, l'adoption, quand elle est formalisée, reste en Angleterre une activité largement privée, menée par des bénévoles au sein d'associations caritatives - ce qui contraste avec l'interventionnisme de l'État français 10 -, à partir de l'après-guerre elle, est peu à peu supervisée par les collectivités locales et menée par des professionnels : les travailleurs sociaux des agences publiques d'adoption. L'Adoption of Children (Regulation) Act de 1939, qui ne commence à être appliqué qu'aprèsguerre, et l'Adoption of Children Act de 1949 placent les acteurs privés de l'adoption sous l'autorité des collectivités locales. Hormis cette évolution, le régime juridique de l'adoption en Angleterre-Galles reste globalement le même jusqu'à l'Adoption Act de 1976.

En France, l'adoption plénière est introduite par le décret-loi du 29 juillet 1939, aussi appelé Code de la famille. Suite à la Première Guerre mondiale et à la loi du 19 juin 1923,

9. Jean-Marc RoHRBASSER, «Hécatombe », in ID. (dir.), Bouleversements démographiques de la Grande Guerre, Paris : INED, 2014, p. 9-18.

10. Pierre LAROQue et Allen Daly, Les travailleurs sociaux en France et en Angleterre. Enquête sur le personnel social et médico-social familial dans les deux pays, Genève: OMS, 1956; et Anne DAGUERRE, La protection de l'enfance en France et en Angleterre, 1980-1989, Paris : L'Harmattan, 1999. 
l'adoption pouvait porter sur des mineurs, mais elle restait une adoption simple. Ce Code de la famille de 1939 comporte plusieurs dispositions importantes. D'une part, un mineur ne peut devenir adoptable que s'il est orphelin ou abandonné et âgé de moins de cinq ans. D'autre part, peuvent adopter des couples mariés depuis plus de dix ans, dont au moins un membre a plus de quarante ans, et qui sont sans enfant légitime. Enfin, l'adoption (appelée jusqu'en 1966 «légitimation adoptive ») substitue à l'ancien lien de filiation entre l'adopté et sa famille d'origine un nouveau lien, et l'adopté hérite du nom et du patrimoine de l'adoptant comme le ferait un enfant légitime. De même qu'en Angleterre-Galles, le régime juridique de l'adoption est peu modifié jusqu'à la loi du 11 juillet 1966.

\section{I.3. Depuis le tournant des années 1960 et 1970}

En Angleterre-Galles aussi bien qu'en France, les années 1960 et 1970 sont marquées par la libéralisation de la contraception et de l'interruption volontaire de grossesse, ainsi que par l'atténuation du stigmate dont sont l'objet les enfants illégitimes et leurs mères (ce dont témoigne l'introduction d'aides publiques destinées aux familles monoparentales). Ces tendances, en diminuant le nombre de conceptions et de naissances non désirées mais aussi en atténuant la honte d'être «fille-mère », réduisent le nombre de mineurs et surtout de nouveau-nés qui sont confiés à l'adoption. C'est dans ce contexte, et suivant une volonté croissante de protéger et promouvoir le seul «intérêt supérieur de l'enfant » (the best interests of the child), que les législateurs français et britannique modifient plusieurs dispositions sur l'adoption plénière, sans toutefois remettre en cause son organisation générale.

En France, l'adoption est réformée par la loi du 11 juillet 1966, qui précise les conditions de l'adoption plénière, notamment en clarifiant l'établissement de la situation d'abandon des mineurs et donc leur adoptabilité. Et alors que, jusqu'en 1976, les adoptants devaient être sans enfant légitime afin que l'adoption ne lèse pas ces enfants, depuis la loi du 22 décembre 1976, les parents sont autorisés à adopter. D'un point de vue procédural, depuis les lois du 6 juin 1984 et du 25 juillet 1985, ne peuvent adopter que ceux qui disposent de l'agrément pour adopter, qui vise à garantir que les adoptants ont les aptitudes matérielles, psychologiques et éducatives pour être de bons parents et promouvoir l'intérêt et le bien-être de l'adopté.

En Angleterre-Galles, l'adoption est réformée par l'Adoption Act de 1976. Comme en France, cette loi précise les conditions de l'adoption plénière : pour qu'un enfant soit placé en vue de l'adoption, il faut soit que chaque parent ou gardien de l'enfant consente à l'adoption (plus de six semaines après la naissance), soit qu'un tribunal ait jugé, dans l'intérêt de l'enfant, qu'aucun parent n'est connu ou ne peut donner son consentement ou que les parents ont négligé ou gravement maltraité l'enfant. Cette loi dispose aussi qu'un candidat à l'adoption doit s'adresser à une agence d'adoption, sauf s'il est apparenté au futur adopté ${ }^{11}$. L'importance des agences privées d'adoption dans le système anglais s'est réduite à partir des années 1970 : elles ne participent plus qu'à un tiers des adoptions dans les années 1990. La loi, renforcée par le Children Act de 1989, dispose aussi que les tribunaux décident de l'adoption dans le seul intérêt de l'enfant, en prenant en considération « ses désirs et ses sentiments » compte tenu « de son âge et de sa compréhension ». L'Adoption Act de 1999, quant

11. Sur les associations privées à but non lucratif agréées par le ministère de la Santé et les agences d'adoption publiques des collectivités locales, voir Laurence BAQUE, « Le rôle des services et agences d'adoption en Angleterre », in Agnès FINE et Claire NEIRINCK (dir.), Parents de sang, parents adoptifs, op. cit., p. 103-116. 
à lui, modifie certaines dispositions applicables à l'adoption internationale, afin que le Royaume-Uni puisse ratifier la Convention de La Haye sur la protection des enfants et la coopération en matière d'adoption internationale. L'Adoption and Children Act de 200212 et l'Adoption and Children Act de 2014, qui remplacent l'Adoption Act de 1976, sont les dernières lois qui modernisent le droit britannique de l'adoption nationale et internationale, dans le but d'augmenter le taux d'adoption des mineurs pris en charge par les services sociaux. Pour pouvoir adopter, il faut désormais être une personne majeure de 21 ans agissant seule ou encore un couple marié ou non et formé de personnes de sexes différents ou de personnes de même sexe, pourvu que les partenaires soient engagés dans une relation familiale de long terme.

\section{I.4. L'état actuel du droit de l'adoption plénière}

Actuellement, les droits français et britannique de l'adoption plénière sont donc relativement proches. D'une part, le principal objectif de l'adoption est le même: promouvoir l'intérêt de l'enfant adopté. Au fil du temps, on a observé la même évolution : pour le législateur, l'adoption ne vise plus véritablement à satisfaire l'intérêt de l'adoptant (élever un enfant), ni a fortiori celui des parents d'origine de l'adopté (les dispenser d'élever leur enfant), mais à répondre à l'intérêt de l'adopté lui-même (lui donner des parents aimants). Si aujourd'hui les pouvoirs publics cherchent à augmenter le taux (et raccourcir le délai) d'adoption des mineurs nationaux adoptables, c'est au motif que tel est l'intérêt des mineurs. Dans les deux pays, cet objectif butte toutefois sur l'importance numérique des mineurs dits à «besoins spécifiques » (special needs), c'est-à-dire âgés, en fratrie ou handicapés, qui sont relativement difficiles à placer pour adoption.

\section{Tableau 1 \\ Le droit de l'adoption plénière en France et en Angleterre-Galles en 2015}

12. Katherine O’Donovan, «L'adoption dans le droit du Royaume-Uni », Revue internationale de droit comparé, 55 (4), 2003, p. 845-860. 


\begin{tabular}{|c|c|c|c|}
\hline & & France & Angleterre-Galles \\
\hline \multirow{4}{*}{ 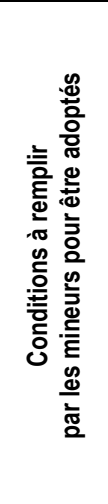 } & $\begin{array}{l}\text { Âge et statut matri- } \\
\text { monial }\end{array}$ & $\begin{array}{c}\text { Être mineur de } 15 \text { ans, } \\
\text { et avoir au moins } 15 \text { ans de moins } \\
\text { que l'adoptant }\end{array}$ & $\begin{array}{c}\text { Être célibataire } \\
\text { et mineur de } 18 \text { ans }\end{array}$ \\
\hline & $\begin{array}{l}\text { Perte de lien de } \\
\quad \text { filiation }\end{array}$ & $\begin{array}{l}\text { Être sans famille (« pupille de } \\
\text { l'État » ou déclaré abandonné } \\
\text { par jugement du tribunal), ou avoir } \\
\text { été expressément consenti } \\
\text { à l'adoption par ses parents }\end{array}$ & $\begin{array}{c}\text { Être déclaré adoptable } \\
\text { par jugement du tribunal } \\
\text { ou avoir été consenti } \\
\text { à l'adoption par ses parents }\end{array}$ \\
\hline & Consentement & $\begin{array}{c}\text { Consentement nécessaire si } \\
\text { l'adopté } \\
\text { a plus de } 13 \text { ans }\end{array}$ & - \\
\hline & $\begin{array}{l}\text { Durée de placement } \\
\text { dans la future } \\
\text { famille }\end{array}$ & Au moins 6 mois & Au moins 12 mois \\
\hline \multirow{2}{*}{ 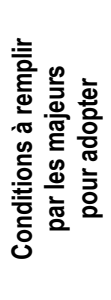 } & $\begin{array}{l}\text { Âge et statut matri- } \\
\text { monial }\end{array}$ & $\begin{array}{l}\text { Être majeur de } 28 \text { ans, ou être } \\
\text { un couple marié depuis plus de } 2 \text { ans } \\
\text { ou dont les deux membres } \\
\text { ont plus de } 28 \text { ans }\end{array}$ & $\begin{array}{l}\text { Être majeur de } 21 \text { ans, ou être } \\
\text { un couple (marié ou non) dont les } \\
\text { deux membres ont plus de } 21 \text { ans }\end{array}$ \\
\hline & Agrément & $\begin{array}{c}\text { Nécessaire, il est accordé } \\
\text { par le service départemental de } \\
\text { l'Aide sociale à l'enfance } \\
\text { pour cinq ans }\end{array}$ & $\begin{array}{l}\text { Nécessaire, il est accordé } \\
\text { par l'agence d'adoption }\end{array}$ \\
\hline \multirow{2}{*}{$\begin{array}{l}\text { 음 } \\
\text { 응 } \\
\text { 응 } \\
\text { 일 } \\
\text { 윯 } \\
\text { 옴 }\end{array}$} & Administrative & $\begin{array}{l}\text { L'Aide sociale à l'enfance, } \\
\text { qui recueille les mineurs adoptables, } \\
\text { apparente le mineur adoptable et } \\
\text { son adoptant }\end{array}$ & $\begin{array}{l}\text { L'agence d'adoption, qui peut être } \\
\text { une agence publique locale } \\
\text { ou une agence caritative privée } \\
\text { agréée et qui recueille les mineurs } \\
\text { adoptables, apparente le mineur } \\
\text { adoptable et son adoptant }\end{array}$ \\
\hline & Judiciaire & $\begin{array}{l}\text { Le jugement d'adoption } \\
\text { est prononcé par un tribunal } \\
\text { (tribunal de grande instance) }\end{array}$ & $\begin{array}{l}\text { Le jugement d'adoption est } \\
\text { prononcé par un tribunal } \\
\text { (family court) }\end{array}$ \\
\hline \multirow{2}{*}{ 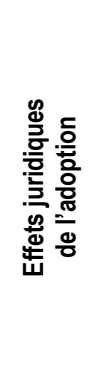 } & Droit de la filiation & $\begin{array}{l}\text { L'adoptant et l'adopté ont le même } \\
\text { lien de filiation qu'un parent } \\
\text { et son enfant légitime. } \\
\text { L'adoption est irrévocable }\end{array}$ & $\begin{array}{l}\text { L'adoptant et l'adopté ont le même } \\
\text { lien de filiation qu'un parent et son } \\
\text { enfant légitime. L'adoption est } \\
\text { irrévocable, sauf exceptions }\end{array}$ \\
\hline & Droit social & $\begin{array}{l}\text { Les adoptants bénéficient } \\
\text { de la prime à l'adoption ou de la } \\
\text { prestation d'accueil du jeune } \\
\text { enfant et, s'ills sont salariés, d'un } \\
\text { congé d'adoption de même durée } \\
\text { que les congés de maternité } \\
\text { postnatal et de paternité }\end{array}$ & $\begin{array}{l}\text { Les adoptants salariés bénéficient } \\
\text { d'un congé d'adoption (adoption } \\
\text { leave) et peuvent bénéficier } \\
\text { de prestations lorsque l'adopté } \\
\text { a des besoins spécifiques } \\
\text { (adoption allowances) }\end{array}$ \\
\hline
\end{tabular}

D'autre part, les dispositions du droit de l'adoption sont très proches en France et en Angleterre-Galles (voir tableau 1, qui ne concerne que l'adoption extrafamiliale, l'adoption intrafamiliale et notamment celle du bel-enfant étant soumise à moins de conditions). Il faut dire que les deux pays ont ratifié la Convention de La Haye (la France en 1998, l'Angleterre- 
Galles en 2003) ${ }^{13}$ et modifié leurs droits respectifs de l'adoption internationale - voire nationale - en conséquence.

$\mathrm{Au}$ regard du droit anglais, certaines dispositions du droit français apparaissent difficilement compréhensibles. Pourquoi la France n'autorise-t-elle pas l'adoption par des concubins (de sexes opposés), alors qu'elle autorise l'adoption par une personne seule et qu'elle ouvre l'aide médicale à la procréation aux concubins de sexes opposés qui sont en couple depuis au moins deux ans ${ }^{14}$ ? La principale différence entre les droits français et britannique en matière d'adoption plénière porte sur la possibilité qu'ont les adoptés de rechercher leurs origines, c'est-à-dire d'obtenir sur celles-ci des informations non identifiantes (état de santé des géniteurs, raisons et circonstances de l'abandon) voire des informations identifiantes (identité de la mère, du père, des ascendants, descendants et collatéraux). En Angleterre-Galles, les adoptés disposent d'un droit à connaitre leurs origines, ce qui n'est pas le cas en France. Tout d'abord, en droit français, depuis la création de l'état civil en 1792, une mère peut légalement ne pas mentionner son nom sur l'acte de naissance de son enfant biologique, alors qu'en droit anglais elle n'a pas cette possibilité. En conséquence, les enfants adoptés après avoir été abandonnés à la naissance peuvent plus aisément connaître leurs origines en Angleterre-Galles qu'en France.

Ensuite, même si dans les deux pays l'adoption donne lieu à un nouvel acte de naissance (birth certificate), en droit français il est interdit de consulter l'acte de naissance originel d'un adopté, alors qu'en droit anglais l'adopté (et lui seul) peut, depuis l'Adoption Act de 1976, consulter son acte de naissance originel dès qu'il est devenu majeur ${ }^{15}$. Cet acte de naissance originel, qui peut être obtenu auprès du registre des adoptions du greffe central de l'état civil (Adopted Children Register du General Register Office), contient la date et le lieu de naissance de l'adopté, son nom de famille à sa naissance, le nom de sa mère, le nom de son père s'il a été inscrit, et le nom de la personne qui a fait établir son acte de naissance. En outre, depuis le Children Act de 1989, les adoptés devenus majeurs et leurs parents biologiques peuvent, si les deux parties y consentent, s'échanger des informations, identifiantes ou non, par le biais d'un registre de mise en contact (Adoption Contact Register) et d'autres institutions (intermediary agencies).

Enfin, depuis l'Adoption and Children Act de 2002, pour retrouver ses parents biologiques, l'adopté peut consulter non seulement son acte de naissance originel mais aussi les informations identifiantes que détiennent les agences d'adoption. En France, ce n'est que depuis la loi du 22 janvier 2002 que les adoptés devenus majeurs et leurs parents biologiques peuvent, d'un commun accord, s'échanger des informations par le biais d'une instance de médiation, le Conseil national pour l'accès aux origines personnelles. Mais les personnes nées dans le secret («sous X ») n'ont pas de droit à connaître leurs origines : si leur mère biologique continue de refuser de révéler certaines informations et a fortiori son identité, elle ne peut être contrainte de le faire, ce que de nombreux observateurs anglo-saxons considèrent comme une violation de la Convention internationale des droits de l'enfant (1989), qui dis-

13. Conférence de La Haye de droit international privé, « Texte et signataires », 2014. Disponible à l'adresse suivante : <http://www.hcch.net/index_fr.php ?act=conventions.status\&cid=69>.

14. Pierre MuRAt, «Les transformations de la famille. Quel impact sur les finalités de l'adoption?», Informations sociales, 146 (2), 2008, p. 20-33.

15. En Angleterre-Galles, jusqu'aux années 1970, les adoptés ne peuvent pas rechercher leurs origines : on craint alors que la génitrice n'interfère dans la vie de la famille adoptive et que le caractère illégitime de l'enfant soit connu et que cela lui nuise. 
pose que «l'enfant est enregistré aussitôt sa naissance et a dès celle-ci le droit à un nom, le droit d'acquérir une nationalité et, dans la mesure du possible, le droit de connaitre ses parents et d'être élevé par eux » (article 7).

\section{Des adoptés plus nombreux en Angleterre-Galles qu'en France jusqu'aux années 2000}

Depuis que les droits français et anglais connaissent l'adoption plénière, comment les comportements d'adoption ont-ils évolué ? Nous comparons ici les nombres d'adoptions entre les deux pays puis, dans la partie suivante, les profils des adoptés.

\section{Les sources statistiques sur l'adoption en France et en Angleterre-Galles}

La France et l'Angleterre-Galles sont deux des rares pays dans lesquels existent des données statistiques de relativement long terme sur l'adoption. En effet, dans ces deux pays, une administration centralisée recense chaque année les adoptions, et décrit parfois certaines des caractéristiques des adoptés et/ou des adoptants. Ces données sont d'autant plus précieuses que, les chercheurs n'ayant généralement pas accès aux dossiers d'adoption, elles sont le seul élément empirique qui permet de décrire l'adoption sur le long terme. Notons toutefois qu'aucune source ne renseigne sur l'évolution du profil des femmes ou des couples qui ont confié leur enfant à l'adoption. Il s'agit là de l'aspect le plus méconnu de l'histoire de l'adoption, aussi bien en France et en AngleterreGalles que dans la plupart des autres pays.

En France, la principale source d'information est le Compte général de l'administration de la Justice civile et commerciale et de la Justice criminelle de 1933 à 1960, le Compte général de l'administration de la Justice criminelle et de la Justice civile et commerciale de 1961 à 1976, puis l'Annuaire statistique de la Justice depuis 1981. Il s'agit d'un annuaire du ministère de la Justice qui recense les jugements d'adoption depuis 1952 (les données sont indisponibles de 1939 à 1951). Cette publication ne comportant plus d'informations sur l'adoption depuis le début des années 1980, elle est complétée par deux enquêtes ponctuelles que le ministère de la Justice a réalisées sur les années 199216 et 200717.

En Angleterre-Galles, la principale source est l'Adoptions in England and Wales 18 . Il s'agit d'une publication annuelle de l'Office for National Statistics, qui recense les jugements d'adoption (adoption orders) depuis 1927. Toutefois, ces données du registre des adoptions n'incluent que les adoptions nationales (extra- et intrafamiliales), et non pas les adoptions internationales (adoptions par les résidents en Angleterre-Galles de mineurs nés puis adoptés à l'étranger). Mais, comme nous le verrons, cela ne modifie aucunement les ordres de grandeur ni les évolutions observés.

Depuis que des données comparables sont disponibles, le nombre d'adoptés en AngleterreGalles a toujours été plus élevé que celui d'adoptés pléniers en France (voir graphique 1). Du début des années 1950 à la fin des années 1970, l'écart est même impressionnant : chaque année, de 2 à 15 fois plus de mineurs sont adoptés en Angleterre-Galles qu'en France. Depuis les années 1980 et plus encore les années 2000, cet écart s'est fortement réduit : de nos jours, en France comme en Angleterre-Galles, on compte chaque année environ 4000 à 5000 adoptés.

\section{Graphique 1 \\ Nombre annuel d'adoptés en France et en Angleterre-Galles, 1927-2012}

\footnotetext{
16. Zakia BelmoKhtAR, « Les adoptions simples et plénières en 1992 », Infostat Justice, 46, 1996, p. 1-4.

17. ID., « L'adoption simple et plénière en 2007 : des projets différents », Infostat Justice, 106, 2009, p. 1-6.

18. OfFice For NAtional Statistics, «Adoptions in England and Wales», 2014. Disponible à l'adresse suivante : <http://www.ons.gov.uk/ons/rel/vsob1/adoptions-in-england-and-wales/index.html>.
} 


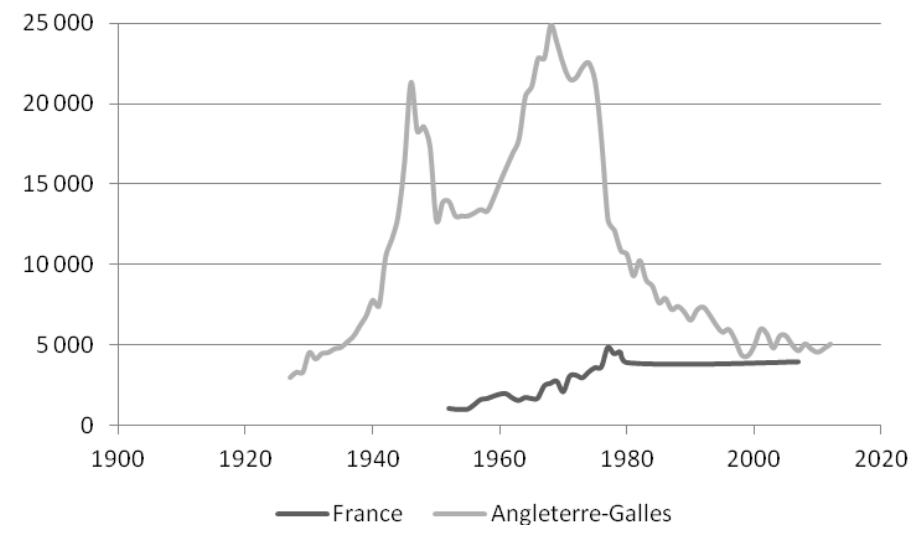

Champs : en France, il s'agit des mineurs adoptés pléniers nationaux (beaux-enfants, très minoritaires, inclus) et internationaux dont le jugement d'adoption a été prononcé par un tribunal de première instance / grande instance, par année de jugement d'adoption ; en Angleterre-Galles, il s'agit des mineurs adoptés nationaux (beauxenfants inclus), par année d'inscription au registre des enfants adoptés (les données d'adoptés nationaux par année de jugement d'adoption sont inexistantes avant 1998, et les données d'adoptés internationaux ne sont pas disponibles sur le long terme mais leur effectif est inférieur à 400 par an sur toute la période).

Source des données : MINISTERE DE LA JUSTICE, Compte général de l'administration de la Justice civile et commerciale et de la Justice criminelle, Paris : Ministère de la Justice, 1952-1960 ; ID., Compte général de l'administration de la Justice criminelle et de la Justice civile et commerciale, Paris : Ministère de la Justice, 1961-1976 ; ID., Annuaire statistique de la Justice, Paris : Ministère de la Justice, 1980 ; Zakia BELMOKHTAR, « Les adoptions simples et plénières en 1992 », art. cité, p. 1-4 ; ID., "L'adoption simple et plénière en 2007 : des projets différents », art. cité, p. 1-6 ; OfFICE FOR NATIONAL StATISTICS, « Adoptions in England and Wales », 2014.

L'évolution du nombre annuel d'adoptés en Angleterre-Galles est particulièrement intéressante, puisqu'elle révèle plusieurs périodes de l'histoire démographique anglaise depuis l'entredeux-guerres. Outre que le nombre d'adoptés croît au fil des années 1930, il connaît un pic très élevé pendant la Seconde Guerre mondiale puis après-guerre (21 280 adoptés en 1946), sans doute en raison d'un surcroît d'orphelins de guerre mais aussi de naissances illégitimes qui accompagne la plupart des conflits armés (ainsi que du surcroît de beaux-enfants adoptés consécutif au pic de divorces après-guerre). Le nombre d'adoptés augmente de nouveau, fortement, pendant les années 1960, en bonne partie parce que les naissances illégitimes deviennent plus nombreuses mais donnent toujours aussi fréquemment lieu à des abandons de nouveau-nés. Puis, à partir de la fin des années 1960 et du début des années 1970, le nombre d'adoptés chute très fortement : la libéralisation de la contraception et de l'interruption volontaire de grossesse (IVG) et la baisse du stigmate associé aux naissances illégitimes réduisent le nombre de nouveau-nés confiés à l'adoption 19. En outre, ces évolutions sociodémographiques affectant le nombre d'adoptés extrafamiliaux ont été accentuées, depuis le début des années 1950, par l'évolution du nombre de mineurs adoptés par leurs beaux-parents, suite au remariage de leur parent gardien, le

19. Peter Selman, «Patterns of Adoption in England and Wales since 1959 », Social Work Today, 7 (7), 1976, p. 194197. 
plus souvent leur mère ${ }^{20}$. Par contraste, l'évolution du nombre annuel d'adoptés pléniers en France ne concorde pas clairement avec l'histoire démographique française. En effet, outre qu'en France les mineurs adoptés par leur beau-père ne sont que très rarement adoptés en adoption plénière (mais plutôt en adoption simple ${ }^{21}$ ), la grande majorité des adoptés pléniers en France sont nés à l'étranger, si bien que leur nombre est moins sensible à la conjoncture démographique française.

L'écart historique du nombre d'adoptés en France et en Angleterre-Galles pourrait s'expliquer de plusieurs façons. En effet, l'adoption plénière du bel-enfant mise à part, pour qu'une adoption ait lieu il faut que surviennent trois événements consécutifs : tout d'abord, qu'un enfant, le plus souvent illégitime, naisse ; ensuite, qu'il soit orphelin ou abandonné ou retiré à ses parents et placé pour adoption; et enfin, qu'il soit adopté. Ainsi, les variations de nombre d'adoptés peuvent en principe s'expliquer par trois facteurs majeurs.

Premièrement, comme l'Angleterre-Galles a le plus souvent connu un nombre de naissances hors mariage inférieur à celui observé en France (voir graphique 2), on ne peut clairement pas expliquer le surcroit d'adoptions en Angleterre-Galles par une augmentation des naissances hors mariage. S'il est vrai que ces naissances sont plus nombreuses en Angleterre-Galles qu'en France de 1962 à 1971, même sur cette courte période, le surcroît de naissances hors mariage observé en Angleterre-Galles par rapport à la France (qui va de 3000 à 18000 par an) ne peut jamais expliquer la totalité du surcroît d'adoptés (qui va de 15000 à 20000 par an).

Deuxièmement, comme le taux d'adoption des mineurs adoptables (placement order granted) est, aujourd'hui au moins, plus élevé en Angleterre-Galles qu'en France (voir tableau 2), on peut en principe expliquer une partie du surcroît d'adoptions en Angleterre-Galles par un plus fort taux d'adoption des mineurs placés. Alors que le taux d'adoption des mineurs adoptables en 2012 est en France de $30 \%$, en Angleterre-Galles il est d'environ $43 \%$. Cela dit, en France comme en Angleterre-Galles, les nourrissons nationaux adoptables et en bonne santé sont fortement demandés, si bien qu'il est peu probable que les taux d'adoption des mineurs adoptables aient été, sur le long terme, fortement différents d'un pays à l'autre.

\section{Graphique 2}

Nombre annuel de naissances hors mariage en France et en Angleterre-Galles, 1900-2013

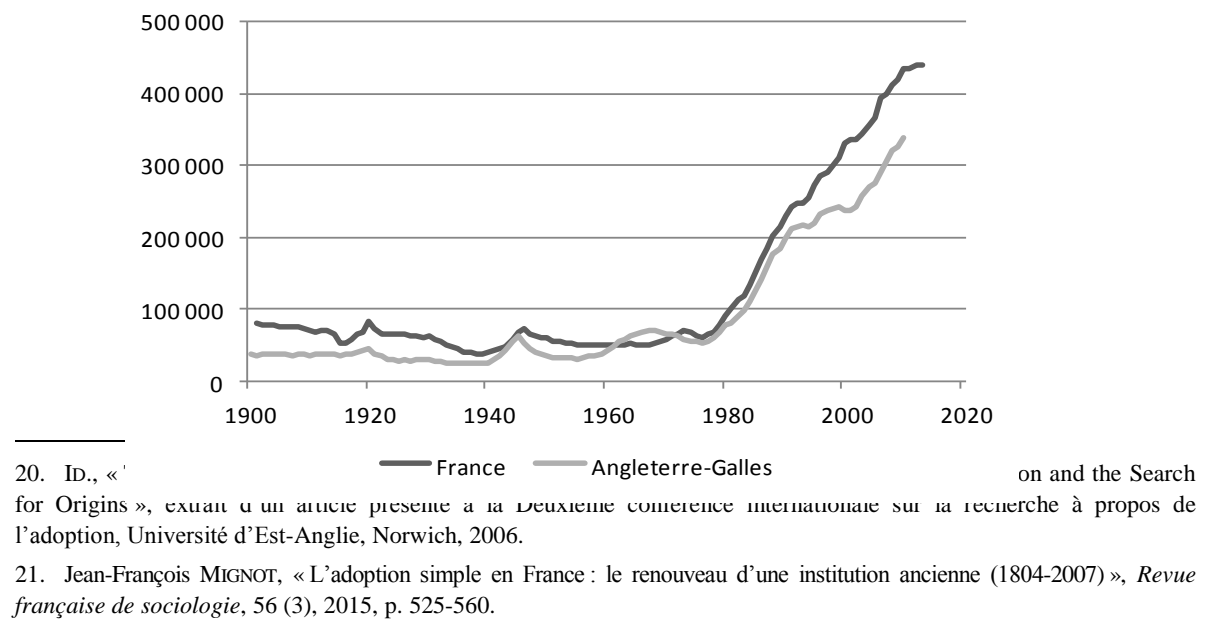


Source des données : Institut national d'études démographiques, base de données des pays développés, disponible à l'adresse suivante : <http://www.ined.fr/fr/pop_chiffres/pays_developpes/base_pays_developpes/>.

\title{
Tableau 2 \\ Les mineurs pris en charge par les services sociaux et adoptables, en France et en Angleterre-Galles en 2012
}

\begin{tabular}{|c|c|c|}
\cline { 2 - 3 } \multicolumn{1}{c|}{} & France * & Angleterre-Galles \\
\hline $\begin{array}{c}\text { « Stock » de mineurs pris en charge } \\
\text { par les services sociaux et adoptables }\end{array}$ & 2328 & $7960^{* *}$ \\
\hline Flux annuel de mineurs adoptés & 731 & $3450^{* * *}$ \\
\hline
\end{tabular}

\begin{abstract}
* OBSERVATOIRE NATIONAL DE L'ENFANCE EN DANGER, « La situation des pupilles de l'État au 31 décembre », rapports 2007-2013.

${ }^{* *}$ Angleterre seulement. SOCIAL AND GENERAL STATISTICS, « Children in Care in England: Statistics », 2014. Disponible à l'adresse suivante : <http://www.parliament.uk/briefing-papers/sn04470.pdf>.

*** DEPARTMENT FOR EDUCATION, «Children looked after in England », 2013. Disponible à l'adresse suivante : $<$ <ttps://www.gov.uk/government/statistics/children-looked-after-in-england-including-adoption>.
\end{abstract}

Nota bene : le « stock » de mineurs est recensé au 31 décembre 2012 en France, et au 31 mars 2012 en AngleterreGalles ; dans chaque pays, le « flux » de mineurs porte sur les 365 jours précédant cette date.

Si l'Angleterre-Galles a longtemps adopté plus voire beaucoup plus de mineurs que la France, c'est sans doute largement pour une autre raison : parce que le taux de placement pour adoption des mineurs nationaux y est bien supérieur. En effet, à partir des années 1950, alors que l'action de protection de l'enfance menée par les travailleurs sociaux se professionnalise, de plus en plus de mineurs sont retirés à leurs parents maltraitants et pris en charge par les services sociaux ${ }^{22}$. Puis une partie, certes minoritaire, de ces mineurs retirés à leurs parents sont placés pour l'adoption, le plus souvent sans l'accord - et même malgré l'opposition - de leurs parents. Par contraste, en France, les pouvoirs publics sont restés largement réticents à placer pour adoption les enfants (même les nourrissons) retirés à leurs parents maltraitants. «Au Royaume-Uni [...], lorsqu'un enfant est pris en charge par les services de protection de l'enfance, un travail est fait avec la famille. Mais si celle-ci ne s'investit pas et se désintéresse de l'enfant, celui-ci est très rapidement proposé à l'adoption. Contrairement à la France, les travailleurs sociaux ne tentent pas de maintenir à tout prix des liens entre l'enfant et ses parents biologiques. Les liens de sang ont beaucoup moins d'importance et les enfants adoptables y sont donc nombreux (plus de cinq mille adoptions nationales par an) ${ }^{23}$. » Ainsi, alors

22. Jenny Keating, A Child for Keeps: The History of Adoption in England, 1918-1945, op. cit.

23. Juliette HALIFAX, L'adoption plénière en France : de l'établissement d'une filiation légale à la constitution d'une filiation sociale, op. cit., p. 120. 
qu'en 2012 on compte, en Angleterre seulement (Pays-de-Galles exclu), 7960 mineurs pris en charge par les services sociaux et en principe adoptables, en France on ne compte que 2328 mineurs adoptables («pupilles de l'État»), soit trois à quatre fois moins (tableau 2). Dans ce contexte, on comprend aisément que le nombre d'adoptions ait longtemps été supérieur en Angleterre-Galles à ce qu'il était en France. Alors qu'en Angleterre-Galles il est relativement fréquent que des mineurs retirés à leurs parents deviennent adoptables, si bien que le «stock » d'adoptables est relativement grand et que les adoptions ont longtemps été nombreuses, en France cette situation reste relativement rare.

\section{Des adoptés nationaux aux profils différents}

Le fait que les mineurs retirés à leurs parents deviennent plus souvent adoptables en Angleterre-Galles qu'en France n'a pas seulement accru le nombre de mineurs adoptables (et adoptés) en Angleterre-Galles. Cela a probablement aussi modifié la composition du groupe des adoptables et de celui des adoptés, même si les données disponibles ne permettent pas toujours de réaliser des comparaisons aussi rigoureuses qu'il serait souhaitable.

Notons tout d'abord qu'en ce qui concerne le sexe et l'âge des adoptables et des adoptés, on ne perçoit ni de différence majeure entre la France et l'Angleterre-Galles, ni d'évolution claire depuis les années $1950 .{ }^{24}$ Ce n'est pas de ce point de vue que les adoptés diffèrent d'un pays à l'autre.

En revanche, la part des adoptés qui sont nés illégitimes a longtemps été beaucoup plus élevée en France qu'en Angleterre-Galles. En France, des années 1950 aux années 1970, la majorité des adoptés pléniers sont des pupilles de l'État, c'est-à-dire des enfants sans famille, pour la très grande majorité nés illégitimes et abandonnés à la naissance. Et, depuis les années 1980, la quasi-totalité des adoptés nationaux restent des pupilles de l'État, nés hors mariage et abandonnés à la naissance ${ }^{25}$. Par contraste, en Angleterre-Galles, la part des adoptés qui sont nés illégitimes n'est que d'environ $55 \%$ dans les années 1970 26. Depuis lors, la part des adoptés qui sont nés hors mariage a augmenté régulièrement pour atteindre $88 \%$ en 2012. En conséquence, alors que les adoptés pléniers nationaux en France ont toujours été, pour la grande majorité d'entre eux, des enfants nés illégitimes et abandonnés dès leur naissance, tel n'est pas le cas en Angleterre-Galles.

De même, alors qu'en France, en 2012, seulement $8 \%$ des mineurs adoptables de l'Aide sociale à l'enfance le sont devenus suite à un retrait total de l'autorité parentale, en Angleterre-Galles, cette part est, vraisemblablement, beaucoup plus importante. Malheureusement, elle n'est pas aisément mesurable : on sait simplement, qu'en 2012, $62 \%$ des mineurs des

\footnotetext{
24 Ministère de la Justice, Compte général de l'administration de la Justice civile et commerciale et de la Justice criminelle, Paris : Ministère de la Justice, 1952-1960; Ministère de la Justice, Compte général de l'administration de la Justice criminelle et de la Justice civile et commerciale, Paris : Ministère de la Justice, 1961-1976; Ministère de la Justice, Annuaire statistique de la Justice, Paris : Ministère de la Justice, 1980 ; Zakia Belmokhtar, « Les adoptions simples et plénières en 1992 », Infostat Justice, 46, 1996, p. 1-4 ; Zakia Belmokhtar, «L'adoption simple et plénière en 2007 : des projets différents », Infostat Justice, 106, 2009, p. 1-6; Office for National Statistics, « Adoptions in England and Wales », 2014.

25. Juliette HaLIFAX, «Grandir hors de sa famille biologique. Cadre de vie des pupilles de l'État et des enfants adoptés », Recherches familiales, 2, 2005, p. 47-67 ; Juliette HALIFAX et Catherine VILLENEUVE-GoKALP, «L'adoption en France : qui sont les adoptés, qui sont les adoptants ?», Population et sociétés, 417, 2005, p. 1-4.

26. Peter Selman, « Patterns of Adoption in England and Wales since 1959 », art. cité, p. 194-197.
} 
services sociaux - adoptables ou non - ont intégré ces services suite à un retrait de l'autorité parentale consécutif à des maltraitances (abuse or neglect), c'est-à-dire des négligences graves et des violences physiques, sexuelles ou psychologiques (cruauté). Quoi qu'il en soit, la part des adoptés qui ont été victimes de maltraitance est sans doute supérieure en Angleterre-Galles (près de $50 \%$ ) à ce qu'elle est en France. Se dessinent ainsi deux profils distincts d'adoptés nationaux. Alors, qu'en France, les adoptés pléniers nationaux sont presque tous nés illégitimes et abandonnés à la naissance, une part importante des adoptés nationaux en Angleterre-Galles sont nés légitimes et/ou n'ont pas été abandonnés par leurs parents : ils leur ont été retirés par les services sociaux, sans ou contre leur avis.

Pour comprendre cette différence majeure entre les deux pays, il convient tout d'abord de rappeler qu'en France, au moins jusqu'aux années 1980, les assistantes sociales ont conçu leur mission de protection de l'enfance comme incompatible avec le signalement de parents maltraitants à la Justice 27. En effet, les travailleurs sociaux craignent que les signalements d'enfants «en danger » conduisent à privilégier leur fonction de «contrôle social » voire de « délation » et de «police morale » des familles de classes populaires dépendantes des aides sociales, au détriment de leur mission de prévention et de protection des mineurs ${ }^{28}$. Ensuite, même si les travailleurs sociaux français hésitent aujourd'hui moins à faire des signalements d'enfants en danger, le débat d'experts et le débat public sur l'opportunité du maintien du lien entre enfant et famille maltraitante témoigne du fait que le législateur, peut-être influencé par des mouvements familialistes, éprouve des réticences à rendre adoptables les enfants maltraités ${ }^{29}$. Par rapport à l'Angleterre-Galles, la France accuse donc un retard marqué en matière de protection de l'enfance ${ }^{30}$, de même qu'en matière de prise en compte de l'identité "ethnoraciale » des parents sur le traitement social de la maltraitance ${ }^{31}$. Cela dit, la position française évolue, et tend actuellement à remettre en cause «le maintien du lien familial à tout prix $\gg 32$.

Quant au profil des adoptants, il diffère assez peu d'un pays à l'autre. En Angleterre-Galles comme en France, depuis l'entre-deux-guerres, la plupart sont des couples mariés, aisés et inféconds. La part des adoptants qui sont des couples mariés atteint $82 \%$ en France en 2007, et $90 \%$ en Angleterre-Galles en 2012. Parmi les adoptants, on a toujours aussi compté quelques personnes agissant seules et qui adoptent leur propre enfant illégitime (afin de leur transmettre leur patrimoine comme à un enfant légitime) ou encore leur bel-enfant ou, plus récemment, des couples de personnes de même sexe. Ainsi, au cours du premier trimestre

27. Delphine SERRE, «La "judiciarisation" en actes. Le signalement d'“enfant en danger" », Actes de la recherche en sciences sociales, 136-137, 2001, p. 70-82 ; ID., «Les assistantes sociales face à leur mandat de surveillance des familles. Des professionnelles divisées », Déviance et société, 34 (2), 2010, p. 149-162.

28. Sur le cas suisse, voir Franz Schultheis, Arnaud FrauENFElder et Christophe Delay, Maltraitance. Contribution à une sociologie de l'intolérable, Paris: L'Harmattan, 2007 ; pour une perspective proche sur le cas britannique, voir John J. Rodger, Family Life and Social Control: A Sociological Perspective, Londres : Macmillan, 1996.

29. Frédéric VABRE, «Le traitement politique de la maltraitance infantile », Recherches et prévisions, 2, 2005, p. 5-16.

30. Frank FuREDI, Paranoid Parenting: Why Ignoring the Experts May Be Best for Your Child, Chicago : Chicago Review Press, 2002.

31. Julia BRophy, «Child Maltreatment in Diverse Households: Challenges to Law, Theory and Practice », Journal of Law and Society, 35 (1), 2008, p. 75-94.

32. Muguette DINI et Michelle MEUNIER, « Rapport d'information fait au nom de la commission des affaires sociales sur la protection de l'enfance », $\mathrm{n}^{\circ} 655$, 2014. Disponible à l'adresse suivante : <http://www.senat.fr/ rap/r13-655/r13-6551.pdf>. 
2013, $6 \%$ des mineurs adoptés en Angleterre-Galles l'ont été par des couples de même sexe 33 .

\section{Des adoptés moins souvent internationaux et des adoptions plus souvent « ouvertes » en Angleterre-Galles qu'en France}

De nos jours, le droit de l'adoption, le nombre annuel d'adoptés et les profils des adoptants sont relativement similaires entre la France et l'Angleterre-Galles. Toutefois, outre le profil des adoptés nationaux, il existe des différences majeures entre l'adoption telle qu'elle est pratiquée dans les deux pays.

\section{IV.1. En l'Angleterre-Galles : très peu de mineurs internationaux adoptés}

Alors que la plupart des adoptés en France sont nés à l'étranger (adoption internationale), la grande majorité des adoptés en Angleterre-Galles sont nés sur place (adoption nationale). Précisément, alors que plus de $70 \%$ des adoptés pléniers en France sont des adoptés internationaux ${ }^{34}$, c'est le cas de seulement $5 \%$ des adoptés en Angleterre-Galles. Il s'agit là d'une différence majeure, qui jusqu'à ce jour n'avait peut-être pas été soulignée à sa juste mesure.

Au sein des pays occidentaux, c'est l'Angleterre-Galles, plutôt que la France, qui apparaît comme un cas «à part » 35 . Alors, pourquoi adopte-t-elle relativement peu de mineurs à l'international ? On peut envisager plusieurs explications. D'une part, il est possible que le nombre relativement élevé de mineurs nationaux qui sont adoptables en Angleterre-Galles permette de satisfaire la demande de la plupart des candidats à l'adoption. À quoi bon adopter par des procédures internationales généralement longues et coûteuses, lorsqu'on réside dans l'un des rares pays occidentaux où il existe des mineurs nationaux adoptables ? Et à quoi bon adopter un enfant de couleur de peau différente, lorsqu'il existe des mineurs adoptables de même couleur de peau que soi (et, s'ils sont âgés, de même langue que la sienne) ? Bref, on peut envisager que si l'Angleterre-Galles adopte si peu à l'étranger, c'est parce que, en rendant adoptables de nombreux mineurs, elle permet par la seule adoption nationale de satisfaire une bonne part de la demande des candidats à l'adoption.

D'autre part, il est probable qu'en Angleterre-Galles le coût de l'adoption internationale soit particulièrement élevé, par rapport à celui de l'adoption nationale. En effet, les services sociaux font payer cher la procédure d'adoption internationale, si bien que le coût total d'une telle adoption s'élève à environ 15000 euros (transports, séjour sur place et frais juridiques sur place inclus), alors qu'une adoption nationale est presque gratuite. Au Royaume-Uni : «Seules les familles les plus riches peuvent accéder à l'adoption internationale. En effet, contrairement à la France, les agréments d'adoption délivrés ne sont pas les mêmes selon que les candidats souhaitent adopter au Royaume-Uni ou à l'étranger et l'agrément pour une adoption internationale coûte extrêmement cher (environ cinq mille livres) ${ }^{36 . » ~ E n ~ o u t r e, ~}$

33. Ministry OF JUSTICE, «Court Statistics Quarterly. January to March 2013 », 2013. Disponible à l'adresse : <https://www.gov.uk/government/uploads/system/uploads/attachment_data/file/207804/court-stats-q1-2013.pdf>.

34. Yves DenEChere, Des enfants venus de loin. Histoire de l'adoption internationale en France, Paris : Colin, 2011.

35. Peter SELman, « Global Trends in Intercountry Adoption : 2001-2010 », Adoption Advocate, 44, 2012 , p. 1-17.

36. Juliette HALIFAX, L'adoption plénière en France : de l'établissement d'une filiation légale à la constitution d'une filiation sociale, op. cit., p. 120-121. Pour quelques éléments empiriques sur les différences de procédure d'adoption interna- 
depuis l'Adoption and Children Act de 2002, si l'adoption internationale est réalisée dans un pays non membre de la convention de La Haye, le jugement d'adoption n'est pas reconnu au Royaume-Uni, si bien que les adoptants doivent rester six mois dans le pays d'adoption avant d'obtenir l'accord des autorités britanniques et qu'un nouveau jugement soit par la suite prononcé au Royaume-Uni. Au coût monétaire s'ajoute alors celui, très élevé, en temps.

Or, il est fort possible que les pouvoirs publics en Angleterre-Galles se satisfassent de cet écart de coût entre adoptions nationales et internationales, puisqu'il incite les candidats à l'adoption à adopter des enfants nationaux, plutôt qu'internationaux, ce qui dispense la collectivité de financer leur recueil. En d'autres termes, en Angleterre-Galles, les pouvoirs publics, confrontés à un nombre élevé de mineurs nationaux adoptables, sont soucieux de l'intérêt de ces mineurs (et des contribuables) ; c'est cela qui les conduit à favoriser largement l'adoption nationale, plutôt qu'internationale. Par contraste, en France, le faible nombre de mineurs adoptables réduit l'intérêt que pourraient avoir les pouvoirs publics à décourager l'adoption internationale au profit de l'adoption nationale.

Enfin, au regard de l'histoire des migrations forcées d'enfants britanniques orphelins ou pauvres vers les colonies canadienne, australienne, néo-zélandaise ou sud-africaine (18601960) ${ }^{37}$, on peut imaginer que les travailleurs sociaux britanniques ont longtemps eu plus de mal que ceux d'autres pays à concevoir que faire voyager un mineur d'un pays à un autre pouvait être dans son intérêt.

\section{IV.2. En France : une adoption « ouverte » très rare}

Alors que la quasi-totalité des adoptions prononcées en France sont «fermées », c'est-àdire qu'elles ne permettent pas à l'adopté d'échanger des informations avec sa famille d'origine, une partie des adoptions prononcées en Angleterre-Galles sont «ouvertes » (open adoption), l'adopté pouvant alors échanger certaines informations avec ses géniteurs. Au sein des pays occidentaux, c'est cette fois-ci la France, plutôt que l'Angleterre-Galles, qui apparaît comme un cas «à part ». En effet, depuis les années 1980, les adoptions «ouvertes » existent aussi aux États-Unis ${ }^{38}$, au Canada 39 ou encore en Allemagne ${ }^{40}$. Cette forme ouverte d'adoption se présente elle-même sous deux modalités, qu'il convient de distinguer, même si elles ont pour point commun d'avoir été consenties par les géniteurs et par la famille adoptive : l'adoption «partiellement ouverte » (semi open adoption), et l'adoption «pleinement ouverte » (full open adoption).

tionale en France et en Angleterre dans les années 1990, voir Joanna GREENFIELD, « Intercountry Adoption: A comparison between France and England », Adoption \& Fostering, 19 (2), 1995, p. 31-36.

37. Joy Melville et Philip BeAN, Lost Children of the Empire, Londres : Unwin Hyman, 1989.

38. Joan Heifetz Hollinger, « L'adoption ouverte aux États-Unis », in Agnès FINE et Claire NEIRINCK (dir.), Parents de sang, parents adoptifs. Approches juridiques et anthropologiques de l'adoption, op. cit., p. $45-61$; Valérie DouMENG, «Étude comparative de l'adoption française et de l'adoption ouverte aux États-Unis », in ibid., p. 147-167; Signe Howell, «Adoption of the Unrelated Child: Some Challenges to the Anthropological Study of Kinship », Annual Review of Anthropology, 38, 2009, p. 149-166. Sur l'histoire mouvementée de l'adoption ouverte aux États-Unis, voir E. Wayne CARP, Family Matters: Secrecy and Disclosure in the History of Adoption, Cambridge : Harvard University Press, 1998.

39. Dominique Goubau, «"Open adoption” au Canada ", in Agnès FINE et Claire NeIRINCK (dir.), Parents de sang, parents adoptifs, op. cit., p. 63-85.

40. Eva WENNER, « Nouvelles formes d'adoption ouverte en Allemagne », in Agnès FINE et Claire NEIRINCK (dir.), Parents de sang, parents adoptifs, op. cit., p. 87-101. 
Dans l'adoption partiellement ouverte, les membres de la famille adoptive n'échangent avec les géniteurs que des informations non identifiantes. Une fois l'adopté devenu majeur, des agences d'adoption ou des officiers d'état civil sont autorisés à consulter l'acte de naissance originel de l'adopté, sans toutefois pouvoir lui communiquer des informations identifiantes sur ses géniteurs. L'adopté peut ainsi connaître certaines caractéristiques de ses géniteurs ou de ses éventuels frères ou sœurs (âge, antécédents médicaux, caractéristiques physiques et identité «ethno-raciale », niveau de diplôme, profession, religion) ou les conditions de son abandon (lieu de naissance, motif de l'abandon, etc.).

Dans l'adoption pleinement ouverte, les membres de la famille adoptive échangent avec les géniteurs des informations identifiantes. Une fois l'adopté devenu majeur, il est autorisé à consulter son acte de naissance originel et/ou son dossier d'adoption, et il peut contacter directement ses géniteurs et ses frères ou sœurs, afin qu'ils puissent s'écrire ou même se voir pour des «retrouvailles » (reunion), de façon ponctuelle ou répétée. Les deux familles peuvent donc s'identifier, afin que les adoptés puissent connaître au mieux leurs origines. Ainsi, en Angleterre-Galles, la plupart des adoptés issus des services sociaux continuent d'être en contact avec leur famille d'origine (birth family contact), non seulement par le biais d'échanges de lettres sans informations identifiantes, mais aussi par des rencontres, sinon fréquentes du moins régulières (une ou deux fois par an).

L'absence d'adoption ouverte en France rend plus difficile celle des enfants maltraités. Mais pourquoi cette forme d'adoption n'existe-t-elle pas en France ? Comme on l'a vu, la France adopte peu de mineurs nationaux (seuls susceptibles d'être adoptés de manière ouverte), et encore la plupart d'entre eux ont-ils été délibérément abandonnés par leur mère ou leurs deux parents à la naissance, si bien qu'ils n'ont jamais eu de liens avec leurs parents biologiques. Dans ce contexte, à quoi bon organiser, par une adoption ouverte, la conservation de liens qui n'ont jamais existé ? Cela ne découragerait-il pas de nombreux adoptants, privant ainsi certains enfants de parents? Par contraste, l'Angleterre-Galles adopte beaucoup de mineurs nationaux qui ont été retirés à leurs parents, parfois à un âge élevé, suite à des mauvais traitements. Dans ce contexte, l'adoption ouverte prend tout son sens : elle permet à des mineurs maltraités de garder contact, dans la mesure où c'est dans leur intérêt, avec des parents dont ils peuvent se souvenir, et qu'ils peuvent encore aimer. En outre, l'adoption ouverte permet aux parents d'origine des adoptés, qui sont le plus souvent défavorables à leur placement pour l'adoption, de garder contact avec leur enfant biologique ; en promettant aux parents maltraitants qu'ils pourront garder contact avec les enfants qui leur sont retirés et qui sont placés pour adoption, l'administration et la Justice respectent les vœux des parents biologiques, ce qui peut rendre plus tolérable une décision à laquelle ils ont le plus souvent été défavorables.

\section{Conclusion}

Comparer l'adoption plénière en France et en Angleterre-Galles est riche d'enseignements. Tout d'abord, alors que les droits de l'adoption de ces deux pays sont, depuis l'entre-deux-guerres, assez similaires, l'Angleterre-Galles a longtemps adopté un nombre beaucoup plus élevé de mineurs que la France. Cela est dû principalement au fait qu'en Angleterre-Galles les mineurs nationaux maltraités sont, beaucoup plus fréquemment qu'en France, placés pour l'adoption, même contre l'avis de leurs parents. Ensuite, depuis les 
années 1970, l'Angleterre-Galles adopte beaucoup moins de mineurs internationaux que la France : les pouvoirs publics sont avant tout soucieux de faire adopter leurs nombreux mineurs nationaux adoptables. Enfin, et toujours depuis les années 1970, l'Angleterre-Galles adopte beaucoup plus par adoption « ouverte » que la France : là encore, il s'agit de promouvoir l'intérêt des mineurs adoptés, dont beaucoup ont été maltraités et peuvent se souvenir de leurs parents d'origine.

Si cet article avait pour ambition de contribuer à la connaissance de l'adoption en Europe, il convient de noter que ce champ de recherche reste peu développé. On ignore encore largement les conséquences à long terme de l'adoption sur le bien-être de l'adopté, en France ${ }^{41}$ comme au Royaume-Uni ${ }^{42}$. Des recherches comparatives seraient pourtant envisageables, puisque, depuis les années 1950, les deux pays ont adopté le même cadre théorique : un attachement «insécure » (insecure) à la mère ou au père, la carence de soins parentaux et a fortiori l'abandon ou la maltraitance sont réputés pouvoir générer divers troubles émotionnels et cognitifs, tels qu'une méfiance systématique à l'égard d'autrui, une difficulté à s'investir émotionnellement dans une relation avec un adulte et des angoisses d'abandon ${ }^{43}$. En outre, on ne sait que très peu de choses sur les effets de chaque système national d'adoption sur le bien-être des adoptés internationaux 44 .

\section{L'auteur}

Chargé de recherche au CNRS et membre du Groupe d'Étude des Méthodes de l'Analyse Sociologique de la Sorbonne (GEMASS), Jean-François Mignot s'intéresse à la démographie et à l'histoire de l'adoption, aussi bien en France et en Europe que dans le monde. Il prépare un ouvrage de synthèse sur ce sujet. Il a récemment publié :

— «L'adoption internationale dans le monde : les raisons du déclin », Population et sociétés, 519, 2015 ;

— «L'adoption simple en France : le renouveau d'une institution ancienne (1804-2007)», Revue française de sociologie, 56 (3), 2015 ;

41. Annick-Camille Dumaret et Marthe Coppel-Batsch, « Effects in Adulthood of Separations and Long-term Foster Care. A French Research Study », Adoption \& Fostering, 22 (1), 1998, p. 31-39 ; Juliette HALIFAX, « L'insertion sociale des enfants adoptés. Résultats de l'enquête "Adoption internationale et insertion sociale" ", Dossiers et recherches de l'Ined, 98, 2000, p. 1-57; Juliette HALIFAX et Marie-Véronique LABASQUE, Étude relative au devenir des enfants adoptés en France et à l'international. Rapport final, Amiens : CREAI de Picardie, 2013.

42. John Triseliotis, Julia Feast et Fiona Kyle, The Adoption Triangle Revisited. A Study of Adoption, Search and Reunion Experiences, Londres : BAAF, 2005 ; Mary MATHER, «Intercountry Adoption », Archives of Disease in Childhood, 92, 2007, p. 479-482 ; Dinithi WiJeDASA et Julie SELWYn, Transition to Adulthood for Adopted Young People: Secondary Analyses of Data from the Longitudinal Study of Young People in England (LSYPE), Bristol : Hadley Trust, 2011 ; Nina BIEHAL, «Outcomes of Adoption: Findings from Research in England », in Quatrième Colloque suisse sur l'adoption internationale, Berne : Bundesamt fur Justiz, 2012, p. 42-44.

43. Gillian SCHOFIELD et Mary BEEK, Attachment Handbook for Foster Care and Adoption, Londres : British Association for Adoption and Fostering, 2006 ; Nathalie SAVARD (dir.), La théorie de l'attachement. Une approche conceptuelle au service de la protection de l'enfance, Paris : ONED, 2010.

44. Joanna GREENFIELD, «Intercountry Adoption: A Comparison Between France and England », art. cité, p. 31-36.

Je tiens à remercier Juliette Halifax, Peter Selman et June Thoburn pour leurs conseils. 
L'adoption plénière en France et en Angleterre-Galles : une histoire comparée du droit et des pratiques (1926-2015)

— « Les adoptions en France et en Italie : une histoire comparée du droit et des pratiques (XIX ${ }^{\mathrm{e}}-\mathrm{XXI}^{\mathrm{e}}$ siècles) », Population, 70 (4), 2015. 